\title{
A short report on Dengue Fever
} Mahwish Fatima, Subia Jamil

\section{- - - - - - - - - - - - - - - - \\ Dear Sir}

Dengue fever is a deadly mosquito-borne viral infection. It is caused by the bite of female mosquito by Aedes aegypti and in some cases also by Aedes albopictus, infected with the virus. Dengue virus is RNA virus, which is single-stranded. There are 4 types of viruses that cause dengue, named DEN-1, DEN-2, DEN-3, and DEN-4. Yet not all four types are found in particular region like Indian sub-continent is mainly restricted by the type 2 and 3. It has been documented that the Pakistan, Sri Lanka, India and Bangladesh are among those countries that are severely affected by the Dengue. In Pakistan, dengue is considered now one of the major health burdens with numerous incidences of complicated cases. ${ }^{1,2}$

People suffering from dengue infection are asymptomatic in $80 \%$ of the cases or present with fever and chills. Some cases come up with the extreme conditions that can be life-threatening to some extent. The incubation period is considered to be 3-14days or usually 4-7days. Abrupt fever, cerebral pain, muscle pain and joint misery are considered the classical manifestations of Dengue fever. Since this condition originates from the muscle and joint pain, it also referred as Break bone fever. Dengue fever course can be categorized into three phases febrile, basic and Recovery.

Diagnosis is based on numerous factors like clinical features, hematological abnormalities and positive IgM antibody test. They all play a significant role in making the diagnosis. Apart from it, virus isolation, viral nucleic acid detection by PCR, virus serotypic/genotypic characterization can be done to confirm the diagnosis. ${ }^{3}$

Currently, no medicines are available for Dengue fever. It has been documented that majority of the patients recover from it. Timely rest, a good proportion of oral fluids and antipyretics show remarkable improvement in out-patients. Yet strong monitoring needs to be done for warning as well as vital signs. Patients, requiring ICU care, blood products, inotropic support and IV fluids are usually in small number. Platelets transfusion seems to be indicated in few cases. Investigation for the use of anti-viral drugs in dengue fever is still in pipe line.,3

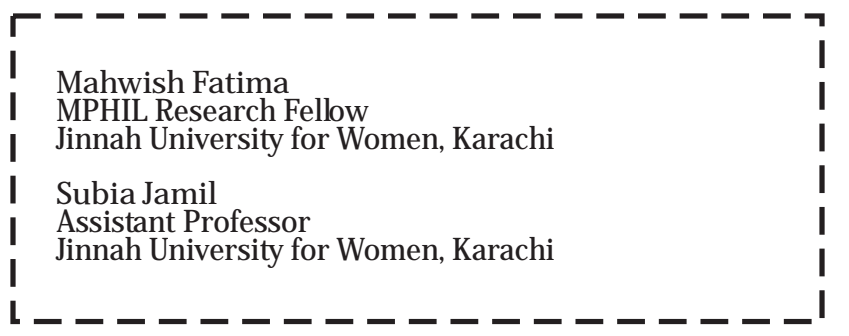

It is strongly needed to isolate vaccines for dengue fever effective against all strains. Presently, after numerous randomized trials, dengue vaccine named, Dengvaxia, (CYD-TDV) has been licensed, yet hasn't approved for use. Moreover, some radical measures to minimize the incidence of dengue fever like mosquito control should be done. Awareness programs should be planned in small areas to create awareness regarding this infection. Misconceptions related to dengue fever have created havoc among people. To address such issues, health care providers need to join their hands at all levels. ${ }^{4}$

Following key points has been documented that claim to be of great help in patients with dengue fever or as preventive measures:

$\varnothing$ Dengue can be suspected in patients having fever more than three days, regardless the presence of other clinical symptoms

$\varnothing$ Avoid prescribing Aspirin/ Ibuprofen to Dengue patients for fever control

$\varnothing$ Go for other laboratory investigation to rule out the cause of fever, if the present syndrome doesn't comply with the Dengue

$\varnothing$ Never go for medicines like steroids, antibiotics or antiviral agents

$\varnothing$ Don't let the serious patient go home, without observing absences of fever for 24-hrs, improving platelet count, overall improvement in appetite and clinical features

$\varnothing$ Don't need to isolate Dengue fever patients. They are not prone to transfer infection to others. All you have to do is to maintain standard precautions like screening hospital windows, and use of mosquito nets in the ward/ room

$\varnothing \quad$ Never go for platelet transfusion or other blood product until find platelet count less than 10,000 and significant bleeding

$\varnothing \quad$ Make sure the use of mosquito repellents, full covered clothes as preventive measures

$\varnothing$ Health awareness program should conduct to minimize the incidence of dengue ${ }^{5}$

\section{REFERENCES:}

1. Mangold KA, Reynolds SL. A review of dengue fever: a resurging tropical disease. Pediatric emergency care. 2013;29(5):665-9.

2. Rasheed S, Butlin R, Boots M. A review of dengue as an emerging disease in Pakistan. Public Health. 2013;127(1):11-7. 
Mahwish Fatima, Subia Jamil

3. Plummer R, Kulkarni R, Sethi A. Dengue fever: the fundamentals. Journal of Pakistan Association of Dermatology. 2016;19(3):127-30.

4. Guzman MG, Harris E. Dengue. The Lancet. 2015; 385 (9966): 453-65.

5. Khan EA. Dengue fever: A major public health problem of our time. Rawal Medical Journal. 2016;41(1).

\section{$\bullet \bullet \bullet$ 个驺}

